\title{
DNA Replication Fading As Proliferating Cells Advance in Their Commitment to Terminal Differentiation
}

SUBJECT AREAS:

DNA REPLICATION

DEVELOPMENT

CELL DIVISION

DNA

Received

11 January 2012

Accepted

30 January 2012

Published

20 February 2012

Correspondence and requests for materials should be addressed to J.B.S. (schvartzman@ cib.csic.es)

\author{
Monturus Ma. Estefanía', Olivier Ganier², Pablo Hernández' ', Jorge B. Schvartzman' ${ }^{1}$, Marcel Mechali² \\ \& Dora B. Krimer ${ }^{1}$
}

'Department of Cell Proliferation \& Development, Centro de Investigaciones Biológicas (CSIC), Ramiro de Maeztu 9, 28040
Madrid, SPAIN, ${ }^{2}$ Institute of Human Genetics, CNRS, 34396 Montpellier, FRANCE.

Terminal differentiation is the process by which cycling cells stop proliferating to start new specific functions. It involves dramatic changes in chromatin organization as well as gene expression. In the present report we used cell flow cytometry and genome wide DNA combing to investigate DNA replication during murine erythroleukemia-induced terminal cell differentiation. The results obtained indicated that the rate of replication fork movement slows down and the inter-origin distance becomes shorter during the precommitment and commitment periods before cells stop proliferating and accumulate in G1. We propose this is a general feature caused by the progressive heterochromatinization that characterizes terminal cell differentiation.

r hroughout the last 2-3 decades significant progress was made in the understanding of how proliferating cells control and regulate initiation and progression of DNA synthesis ${ }^{1-7}$. In contrast, the fading of DNA replication as cells stop proliferating and differentiate, received little or no attention at all. Progressive differentiation of somatic stem cells differs from the terminal differentiation of those cells that, although might be reprogrammed in some cases ${ }^{8}$, are usually not committed to proliferate thereafter. This is one of the most important choices each single cell makes at some point ${ }^{9}$. It is a complex decision that involves dramatic changes in gene expression and chromatin organization ${ }^{2,49}$.

Murine erythroleukemia (MEL) cell lines derive from proerythroblasts transformed with the Friend complex ${ }^{10}$. As in the case of untransformed cells, MEL cells proliferate indefinitely in the absence of erythropoietin. MEL cells may overcome the blockage, however, and reinitiate differentiation when exposed to a number of different chemical agents, such as hexamethylene-bis-acetamide - HMBA. A precommitment period of 12-24 hours, however, is required before cells become irreversibly committed to terminal differentiation ${ }^{10,11}$. In the presence of the inducer MEL cells continue to cycle 4-5 times before proliferation stops and cells accumulate in G1 ${ }^{10}$. These features make MEL cells an invaluable model to study reprogramming of tumour cells to a non-malignant phenotype and to analyse the mode of action of different chemotherapeutic compounds. Some observations indicate that MEL phenotypic differentiation and terminal cell division, however, are not necessarily coupled ${ }^{12}$. Down regulation of genes characteristic of proliferating cells, including several oncogenes such as myc, myb and PU.1, goes along with cell cycle arrest ${ }^{13,14}$. Concomitantly, expression of a number of differentiated cell-gene markers leads to reactivation of the erythroid differentiation program ${ }^{15-18}$.

Here we used cell flow cytometry and genome wide DNA combing to examine for the first time DNA replication during the precommitment and early commitment periods of MEL cells before they stop proliferation and differentiate in the presence of HMBA. The results obtained indicated that replication forks progressively slow down as cells advance in their commitment to differentiate. Concomitantly, the inter-origin distance becomes shorter, indicating that replication origins that were dormant in actively proliferating cells became activated as cells approached terminal differentiation. We confirmed that cells continue cycling for 4-5 rounds in the presence of HMBA, which induced no DNA damage, before proliferation stopped and cells accumulated in G1. In addition, we confirmed that $\mathrm{HP} 1 \alpha$, a marker for heterochromatinization ${ }^{19}$, increases as cells differentiate. As different loci are known to behave disparately during terminal cell differentiation ${ }^{7,20,21}$, these observations strongly suggest that heterochromatinization, which affects most but not all the genome, modulates origin choice and inter-origin spacing during terminal cell differentiation. 


\section{Results}

To confirm that proliferating MEL cells differentiate in the presence of HMBA, samples were taken from three different cultures every 24 hours and cell differentiation was monitored by the benzidine staining reaction. Benzidine reacts with the heme groups of haemoglobin leading to a light blue colour ${ }^{15,17}$. The number of stained cells remained below $1 \%$ in logarithmically growing MEL cells as well as up to 48 hours after the addition of HMBA and increased progressively to over $90 \%$ at 120 hours (Supplementary Figure 1). As cells become irreversibly committed to terminal differentiation 48 hours after exposure to the inducer ${ }^{22}$, we decided to examine DNA replication in cells that were exposed to HMBA for 0, 24 and 48 hours. First, a 20 minutes bromodeuxyuridine (BrdU)-labelling pulse and cell flow citometry was used to determine the distribution of cells along the cell cycle $^{23}$. Figure 1 shows that the number of replicating cells, those cells that incorporated BrdU, progressively dropped from $65.13 \%$ at $0 \mathrm{hr}$ to $42.73 \%$ at $24 \mathrm{hr}$ and $34.48 \%$ at $48 \mathrm{hr}$ in the presence of HMBA. The number of cells in G2/M also dropped from $22.23 \%$ to $17.28 \%$ and $15.09 \%$, respectively. On the other hand, the number of cells in G1 progressively increased from $10.64 \%$ to $36.41 \%$ and $48.33 \%$, respectively. These observations confirmed that although cells continue to cycle after addition of the inducer, they progressively stopped proliferating and accumulated in $\mathrm{G} 1^{10}$.

To determine the rate of DNA replication fork progression and the inter-origin distance genome wide, we used DNA combing and immunocytochemistry. This technique has been successfully used to measure both parameters for several cell types in untreated as well as after cells were exposed to different treatments ${ }^{24-29}$. MEL cells were exposed to two consecutive 20 min pulses with Iododeoxyuridine (IdU) and Chlorodeoxyuridine (CldU) respectively, after they were treated with HMBA for 0, 24 and 48 hours. Selected molecules from this experiment are shown in Figure 2a. Figures $2 \mathrm{~b}$ and $\mathrm{c}$ show the track length of the second (CldU) pulse for molecules that were labelled with both (CldU and IdU) pulses ${ }^{28}$. The most abundant CldU track (corresponding to $25.51 \%$, 62 out of 243 molecules scored) was 30-40 kb long for MEL 0 hours. It dropped to 20$30 \mathrm{~kb}$ long (corresponding to $21.61 \%, 59$ out of 273 molecules scored) for MEL 24 hours and to 10-20 kb long (corresponding to $38.59 \%, 110$ out of 285 molecules scored) for MEL 48 hours. This data clearly showed that the number of molecules with longer track lengths progressively diminished with time, indicating that replication forks slowed down as cells progressed throughout the precommitment and commitment stages of terminal differentiation. Inter-origin distance was measured where CldU tracks were found to flank IdU tracks in two neighbour replicons on the same molecule $^{28}$. Selected molecules corresponding to cells that were labelled 0 , 24 and 48 hours after the addition of HMBA are shown in Figure 3a. Figures $3 b$ and $c$ show the inter-origin distance measured in each case. The most abundant inter-origin distance class (corresponding to $18.24 \%, 29$ out of 159 molecules scored) was $80-100 \mathrm{~kb}$ long for MEL 0 hour. It dropped to $60-80 \mathrm{~kb}$ long (corresponding to $18.01 \%$, 29 out of 161 molecules scored) for MEL 24 hours and to $40-60 \mathrm{~kb}$ long (corresponding to $31.58 \%, 78$ out of 247 molecules scored) for MEL 48 hours. The total number of initiation events per megabase of DNA was 1.39 for MEL 0 hour, 1.40 for MEL 24 hour and 2.10 for MEL 48 hour. It is evident that the inter-origin distance turned progressively shorter as cells advanced throughout the precommitment and commitment stages of terminal differentiation.

We wondered if the response observed was caused by the lack of proliferation by itself and not necessarily associated to differentiation. To test the latter, we first used flow cytometry to check the progression of MEL cells along the cell cycle when they were forced to stop proliferating by serum deprivation. The results obtained are shown in Figure 1d. Contrary to what happened in the presence of $10 \%$ FCS and HMBA (Figure 1c), serum deprivation caused no significant redistribution of cells along the cell cycle compartments. As expected, the total number of cells remained unchanged indicating that cell proliferation has ceased indeed. Despite the abridged incorporation of BrdU, it was sufficient to allow the quantitation of replication fork movement and inter-origin distance after 24 of serum deprivation. After logarithmically growing MEL cells were shifted to a culture medium without FCS for 24 hours they were exposed to two consecutive 20 min pulses with Iododeoxyuridine (IdU) and Chlorodeoxyuridine (CldU) respectively. The rate of DNA replication fork progression and the inter-origin distance genome wide, were determined by DNA combing and immunocytochemistry as described before. The results obtained for the rate of replication fork progression are shown in supplementary figure $3 \mathrm{~A}$. Compared to the data obtained when the cells were grown for 24 hours in the presence of $10 \%$ FCS, the most abundant CldU track remained 20-30 kb long, although its abundance increased from 22 to $33 \%$. The mean track length dropped, though, from 54.03 to $28.89 \mathrm{~kb}$. This is explained by the absence of longer tracks in the sample corresponding to 0\% FCS. Surprisingly, though, the interorigin distance remained almost unchanged (Supplementary figure $3 \mathrm{~B})$. The most abundant inter-origin distance class shifted from $60-80 \mathrm{~kb}$ long for cells grown in the presence of $10 \%$ FCS to $100-$ $120 \mathrm{~kb}$ long in the absence of FCS. The mean inter-origin distance, though, remained almost unchanged $(84.87 \mathrm{~kb}$ for cells grown in the presence of $10 \%$ FCS to $71.77 \mathrm{~kb}$ for cells grown without FCS). Altogether, this data showed that in the absence of FCS the number of molecules with longer track lengths progressively diminished with time, indicating that replication forks slow down. Contrary to what happens as cells differentiate in the presence of HMBA, though, the inter-origin distance showed no significant changes for cells grown without FCS indicating that silent replication origins were not activated when cells stop proliferating due to senescence by itself.

It is well known that nucleotide pools vary along the cell cycle expanding during the S-phase and contracting significantly in $\mathrm{G} 1^{30,31}$. Moreover, the efective concentration of deoxyribonucleoside 5 ' -triphosphates (dNTPs) at sites of DNA replication in vivo are higher than the concentrations of dNTPs averaged over the entire cell volume ${ }^{32}$. For this reason, in order to evaluate dNTPs in asynchronously growing cells nowadays many biochemists prefer to determine the transcription level of ribonucleotide reductase (RNR), the enzyme that catalyses a rate-limiting step in the biosynthesis of all four $\mathrm{dNTPs}^{30,33}$. Here we used real-time PCR to determine the transcription level for the subunit 1 of RNR during HMBA-induced MEL cell differentiation (0, 24 and 48 hours). As a control we used proliferating cells exposed to $2 \mathrm{mM}$ hydroxyurea (HU) for 2 and 18 hours. The results obtained indicated that the levels of RNR-S1 dropped in the presence of HMBA but remained constant after 24 and 48 hours in the presence of the drug (data not shown). On the contrary, RNR-S1 also dropped abruptly after just 2 hours in the presence of HU, but recovered and achieved higher levels after 18 hours. This was probably due to the induction of DNA damage by HU. DNA damage is known to activate RNR to increase the levels of $\mathrm{dNTPs}^{31}$. To check whether or not HMBA also caused DNA damage we used immunocytochemistry to determine the levels of $\gamma \mathrm{H} 2 \mathrm{AX}$ in cells treated with HMBA for 0, 24 and 48 hours. For comparison we used MEL cells exposed to $2 \mathrm{mM}$ HU for 24 and 48 hours as well ${ }^{34}$. The results obtained are shown in Supplementary Figure 2. Although $\gamma \mathrm{H} 2 \mathrm{AX}$ was detected in untreated proliferating cells (MEL $0 \mathrm{hr}$ ), the intensity of the signal remained similar after 24 and 48 hours in the presence of HMBA. On the contrary, the intensity of the signal increased significantly when the cells were cultured in the presence of $\mathrm{HU}^{34}$. These observations confirmed that no DNA damage occurred during the precommitment and commitment periods of differentiating MEL cells in the presence of $\mathrm{HMBA}^{11}$.

\section{Discussion}

In prokaryotes and some unicellular eukaryotes, such as Saccharomyces cerevisiae, it is well established that replication origins are sequence specific, although its nature is less clear in higher eukaryotes ${ }^{1-5}$. 
a
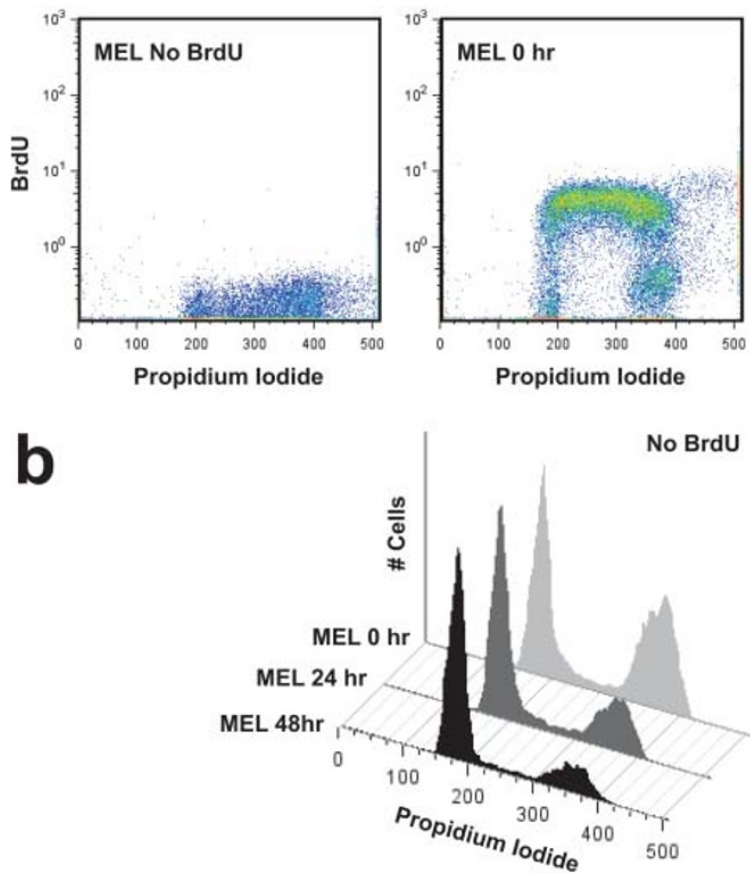
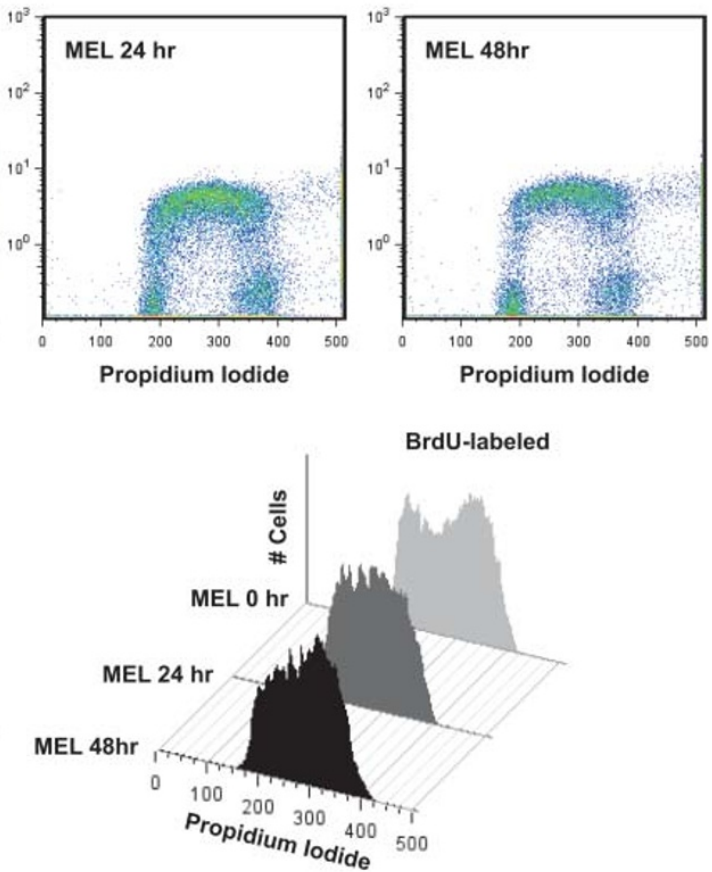

C

Cultured with $10 \%$ FCS \& HMBA

\begin{tabular}{|c|c|c|c|}
\cline { 2 - 4 } \multicolumn{1}{c|}{} & MEL 0 hour & MEL 24 hour & MEL 48 hour \\
\hline S & $65.13 \%$ & $42.73 \%$ & $34.48 \%$ \\
\hline G1 & $10.64 \%$ & $36.41 \%$ & $48.33 \%$ \\
\hline G2/M & $22.23 \%$ & $17.28 \%$ & $15.09 \%$ \\
\hline \# Cells & $3 \times 10^{5}$ & $6 \times 10^{5}$ & $2.14 \times 10^{6}$ \\
\hline
\end{tabular}

d

Cultured without FCS

\begin{tabular}{|c|c|c|c|}
\cline { 2 - 4 } \multicolumn{1}{c|}{} & MEL 0 hour & MEL 24 hour & MEL 48 hour \\
\hline S & $66.44 \%$ & $55.93 \%$ & $65.97 \%$ \\
\hline G1 & $21.30 \%$ & $33.11 \%$ & $26.46 \%$ \\
\hline G2/M & $10.25 \%$ & $10.96 \%$ & $7.53 \%$ \\
\hline \# Cells & $2 \times 10^{5}$ & $2 \times 10^{5}$ & $4.8 \times 10^{5}$ \\
\hline
\end{tabular}

Figure $1 \mid$ Distribution of MEL-DS19 cells throughout the cell cycle during HMBA-induced differentiation. MEL-DS19 cells were pulse-labelled with BrdU, fixed and double stained with propidium iodide (PI) and BrdU-FITC. (a) The upper panels show a bivariate analysis of total DNA content using propidium iodide against BrdU incorporation (BrdU-FITC). Unlabelled cells lie below $10^{\circ}$ of the y-axis while BrdU-labelled cells lie above it. (b) Lower panels show the PI fluorescence distribution of unlabeled (left) or BrdU-labelled (right) nuclei of MEL untreated ( 0 h) or HMBA-treated for 24 or 48 h. Table (c) indicate the calculated percentage of cells in each phase of the cell cycle and the total number of cells per ml at 0,24 and 48 hours, respectively. Finally, table d shows the calculated percentage of cells in each phase of the cell cycle and the total number of cells per ml after the cells were grown without FCS for 0,24 and $48 \mathrm{hr}$. 


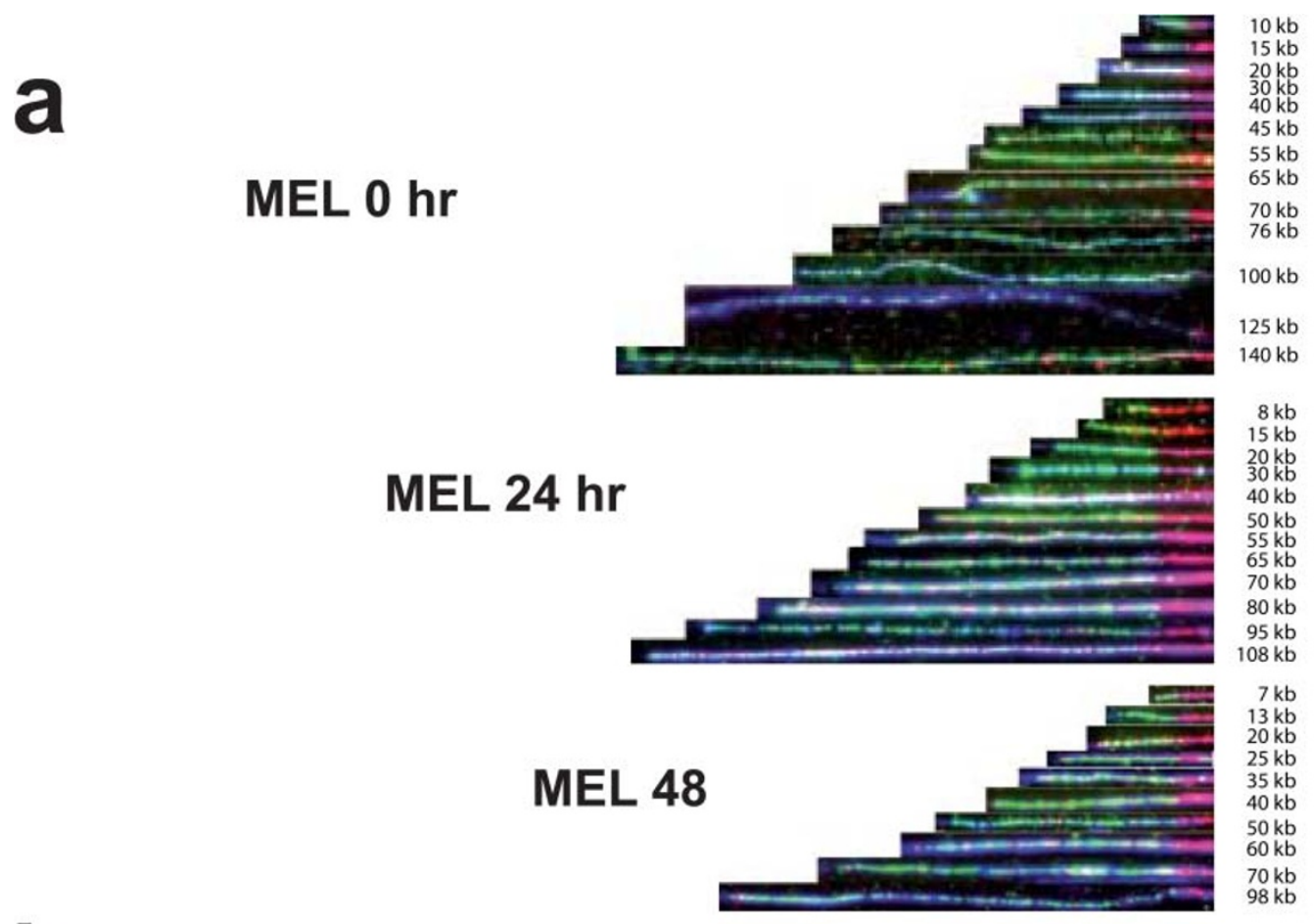

b

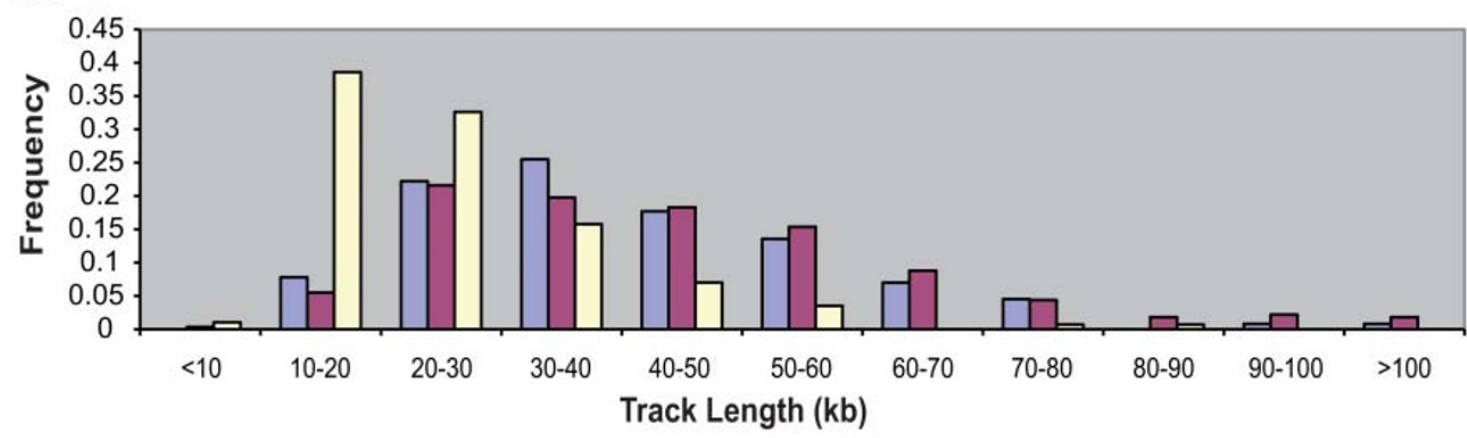

C

CldU Track Length (kb)
$<10$
$10-20$
$20-30$
$30-40$
$40-50$
$50-60$
$60-70$
$70-80$
$80-90$
$90-100$
$>100$

MEL 0

0.00

7.82

22.22

25.51

17.70

13.58

7.00

4.53

0.00

0.82

0.82
MEL 24

0.37

5.49

21.61

19.78

18.32

15.38

8.79

4.40

1.83

2.20

1.83
MEL 48

1.05

38.59

32.63

15.79

7.02

3.51

0.00

0.70

0.70

0.00

0.00

Figure $2 \mid$ The rate of replication fork progression slows down as cells differentiate. MEL-DS19 cells untreated or treated with HMBA for 24 or 48 hr were pulse-labelled for $20 \mathrm{~min}$ with IdU (red) followed by another $20 \mathrm{~min}$ pulse with CldU (green). DNA molecules were stretched by DNA combing as described in Materials and Methods. (a) Representative images of DNA fibres arbitrarily assembled from different fields. Only fibres showing both red and green fluorescence followed by unlabelled DNA (visualized in blue) were measure to guarantee the selection of complete intervals. (b) Distribution of CldU track lengths clustered by frequency measured in $10 \mathrm{~kb}$ intervals for fibres of uninduced MEL (MEL 0) or cells treated with HMBA for 24 (MEL 24 ) or 48 hours (MEL 48). In the histogram, coloured bars point to MEL 0, 24 and 48 from left to right, respectively. P value $<0,0001$ in the Mann Whitney test. The table (c) indicates the calculated percentage of molecules in each interval. 

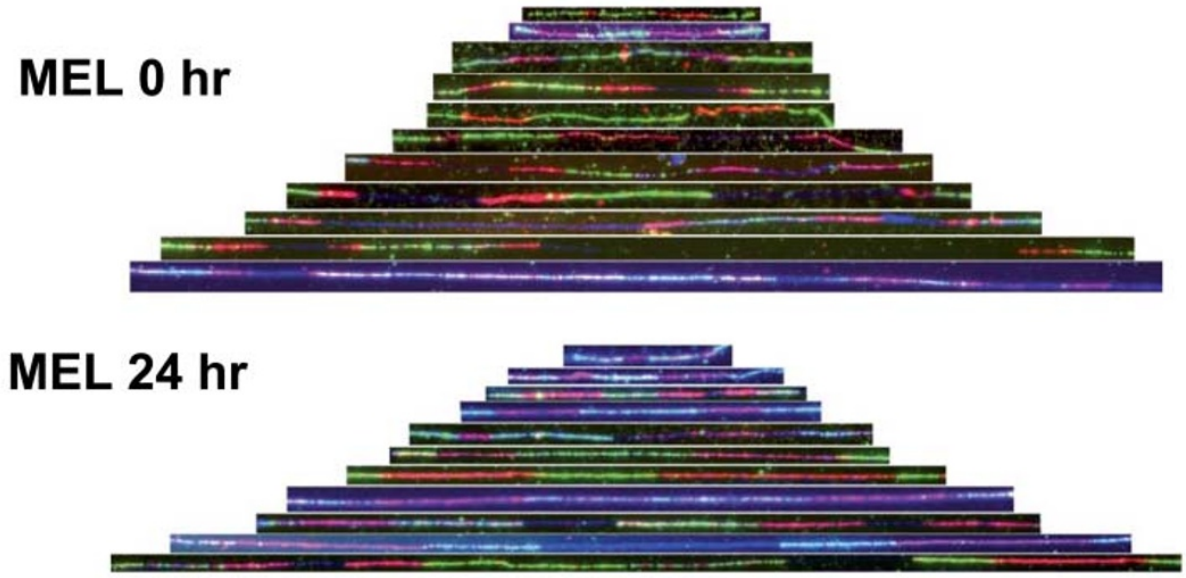

\section{MEL 48 hr}
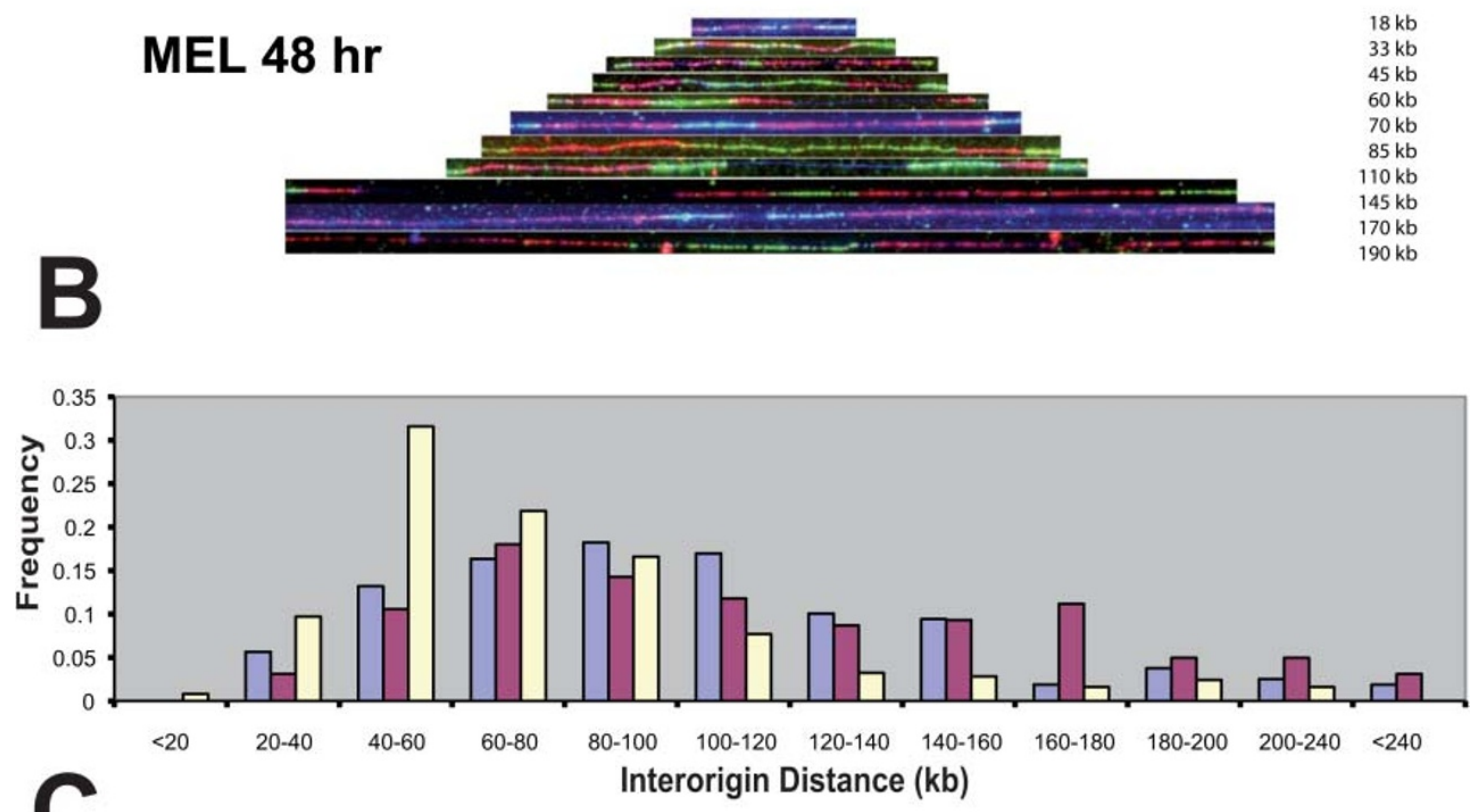

$\begin{array}{crrr}\text { Distance (kb) } & \text { MEL 0 } & \text { MEL 24 } & \text { MEL 48 } \\ \mathbf{<} \mathbf{2 0} & 0,00 & 0,00 & 0,81 \\ \mathbf{2 0 - 4 0} & 5,66 & 3,11 & 9,72 \\ \mathbf{4 0 - 6 0} & 13,21 & 10,56 & 31,58 \\ \mathbf{6 0 - 8 0} & 16,35 & 18,01 & 21,86 \\ \mathbf{8 0 - 1 0 0} & 18,24 & 14,29 & 16,60 \\ \mathbf{1 0 0 - 1 2 0} & 16,98 & 11,80 & 7,69 \\ \mathbf{1 2 0 - 1 4 0} & 10,06 & 8,70 & 3,24 \\ \mathbf{1 4 0 - 1 6 0} & 9,43 & 9,32 & 2,83 \\ \mathbf{1 6 0 - 1 8 0} & 1,89 & 11,18 & 1,62 \\ \mathbf{1 8 0 - 2 0 0} & 3,77 & 4,97 & 2,43 \\ \mathbf{2 0 0 - 2 4 0} & 2,52 & 4,97 & 1,62 \\ \mathbf{2 4 0 - 2 6 0} & 1,89 & 3,11 & 0,00\end{array}$

Figure 3 The interorigin distance turns shorter as cells advance to terminal differentiation. MEL-DS19 cells untreated or treated with HMBA for 24 or $48 \mathrm{hr}$ were pulse-labelled for $20 \mathrm{~min}$ with IdU (red) followed by another $20 \mathrm{~min}$ pulse with CldU (green). DNA molecules were stretched by DNA combing as described in Materials and Methods. (a) Selected DNA molecules isolated from MEL DS19 untreated cells (MEL 0 hr) or treated with HMBA for 24 (MEL $24 \mathrm{hr}$ ) or $48 \mathrm{hr}$ (MEL $48 \mathrm{hr}$ ) containing replication initiation sites. Detection of sequential labelled tracks with IdU (red) followed by CldU (green) and unlabelled (blue) plus subsequent cell harvest guaranteed that the center-to-center distance between adjacent IdU tracks corresponded to neighbour interorigin distances. (b) Distribution of inter-origin distances clustered by frequency measures in intervals of $20 \mathrm{~kb}$ for fibres of uninduced MEL (MEL 0 ) or cells treated with HMBA for 24 (MEL 24) or 48 hours (MEL 48). In the histogram, coloured bars point to MEL 0, 24 and 48 from left to right, respectively (see text for details). P value $<0,0001$ in the Mann Whitney test. The table (c) indicates the calculated percentage of cases in each interval. 
Moreover, several models were proposed suggesting that in these higher eukaryotes initiation of DNA replication could be determined epigenetically, although its regulation is not yet fully understood ${ }^{4,29,35,36}$.

There is increasing evidence indicating that the activation of normally dormant replication origins occurs as a consequence of replication fork slowdown in order to promote complete genome replication ${ }^{35}$. In turn, a decrease in the rate of replication fork movement may have different causes. It was repeatedly shown that in actively proliferating cells nucleotide pool modulates replication fork rate, origin choice and inter-origin spacing, ${ }^{3,2437-43}$. When actively proliferating cells are challenged with suboptimal concentrations of $\mathrm{HU}$, a ribonucleotide reductase inhibitor ${ }^{44}$, the rate of fork movement slows down and replication origins that were silent in unchallenged cells become activated ${ }^{24,37}$. It is unlikely, however, that nucleotide pool alone modulates fork rate movement and inter-origin distance in differentiating cells. Silencing of replication origins has been reported during induced differentiation of mouse P19 cells at a specific locus, the HoxB domain ${ }^{20}$. In this case the nascent strand relative abundance measurement was used to map replication origins. This observation is not in contradiction with the genome wide observations made in the present report as specific loci can behave either way during differentiation $^{21}$. Indeed, evidence for the progressive activation of specific replication origins in the large domains of the immunoglobulin heavy chain locus during B cell development has been reported using the single-molecule analysis of replicated DNA (SMARD) technique ${ }^{7}$. Altogether, these observations challenge the idea that nucleotide pools are the unique modulators for the rate of replication fork movement and origin choice $e^{3,24,37-43}$. In such a case, all loci would be expected to behave alike. Comparison of the results obtained for the estimation of transcription levels for RNR-S1 and DNA damage in cells exposed to HMBA or HU (Supplementary figure 2) and the rate of fork movement and inter-origin distance after serum deprivation (Supplementary figure 3) strengthens this idea.

On the other hand, in Drosophila melanogaster polytene chromosomes, heterochromatic regions are underreplicated, indicating that replication of satellite DNA sequences may represent a barrier for replication ${ }^{45}$. Also, there is evidence suggesting that the rate of replication fork movement differs between euchromatic and heterochromatic zones in mammalian cells ${ }^{46}$. Finally, histone acetylation was found to be a key determinant of chromatin structure. Inhibitors of histone deacetylases change chromatin structure leading to replication fork slowdown and activation of dormant origins ${ }^{25}$. HP1 belongs to a family of proteins containing a motif known as chromodomain that was originally identified as an antigen specifically localized to pericentric heterochromatin in D. melanogaster ${ }^{47}$. In mammalian cells, HP1 is a heterochromatin-associated protein that has a dose-dependent effect on gene silencing and a critical role in heterochromatin formation and maintenance ${ }^{19}$. To determine if heterochromatinization goes along with terminal differentiation in MEL cells, we used immunocytochemistry and flow cell cytometry to check the levels of HP1 $\alpha$ in cells treated with HMBA for 0,24 and 48 hours. The results obtained are shown in Figure $4 \mathrm{a}$ and $\mathrm{b}$. Although HP1 $\alpha$ was evident in proliferating cells (MEL $0 \mathrm{hr}$ ), the $\mathrm{HP} 1 \alpha$-signal increased continuously during the precommitment and commitment periods (MEL 24 and $48 \mathrm{hr}$ ). Flow cell cytometry indicated that the signal increased by a factor of 1.4 in those cells exposed to HMBA for $48 \mathrm{hr}$ (Figure 4b). Altogether, these observations suggest that in differentiating MEL cells the slowdown of replication forks and the consequent activation of dormant origins could be due to the progressive heterochromatinization that characterizes terminal differentiation. Here, it is interesting to note that in welldefined multistep leukemia models, overexpression of Spi-1/PU.1 in mouse and humans shortens S-phase duration by acting specifically on elongation rather than enhancing origin firing ${ }^{48}$. This observation also challenges the idea that dormant origins activation is regulated by neighboring replication fork rates.
In summary, here we used genome-wide analysis of DNA replication to show that in general the rate of replication fork movement slows down and the inter-origin distance becomes shorter during the precommitment and commitment periods of MEL terminal differentiation before cells stop proliferating and accumulate in G1. We propose this is a general feature that occurs as a consequence of the progressive heterochromatinization that takes place during terminal cell differentiation.

\section{Methods}

Cell culture. MEL DS-19 cells were maintained in Dulbecco's modified Eagle's medium DMEM, (Gibco) supplemented with 10\% fetal bovine serum, FBS (Cambrex) and 100 units/ml of penicillin and streptomycin (Gibco). Differentiation was induced by exposing logarithmically growing cultures to $5 \mathrm{mM}$ HMBA. Hemoglobinized cells were monitored by determining the proportion of benzidinestaining cells $(\mathrm{B}+)$ in the culture. Briefly, aliquots $(0.1 \mathrm{ml})$ of culture containing from $10^{4}$ to $10^{5}$ cells were mixed with $0.1 \mathrm{ml}$ of a $77.7 \mathrm{mM}$ benzidine solution and $15 \mu \mathrm{l}$ of $30 \% \mathrm{H}_{2} \mathrm{O}_{2}$. After 20 minutes of incubation at room temperature 500-700 cells per sample were counted with a hemocytometer. To induce quiescence, cells were washed with PBS to completely eliminate fetal bovine serum and maintained in Dulbecco's modified Eagle's medium, DMEM (Gibco), supplemented with 100

units/ml of penicillin and streptomycin (Gibco) for 24 and 48 hours.

BrdU labelling and flow cytometry. MEL DS-19 cultures, $3 \times 10^{5}$ cells $/ \mathrm{ml}$, growing in the absence $(0 \mathrm{hr})$ or in the presence of $5 \mathrm{mM} \mathrm{HMBA}$ ( 24 and $48 \mathrm{hr}$ ) were pulselabelled with $20 \mu \mathrm{M}$ 5-bromo-2' -deoxyuridine (BrdU, Sigma Aldrich) for 20 minutes at $37^{\circ} \mathrm{C}$, fixed with $70 \%$ ethanol and kept overnight at $4^{\circ} \mathrm{C}$. Cells were permeabilized for 30 minutes at room temperature with a solution containing $200 \mu \mathrm{g}$ of pepsin (Sigma Aldrich) in $2 \mathrm{M} \mathrm{HCl}$. After permeabilization, cells were washed three times in PBS at room temperature. The pellet was resuspended in $0.3 \mathrm{ml}$ of PBS supplemented with $0.5 \%$ Tween 20 and $0.5 \%$ BSA, containing $15 \mu \mathrm{L}$ of anti-BrdU-FITC-Ab (Becton Dickinson) for 1 hour at room temperature. Samples were resuspended in PBS, stained with $20 \mu \mathrm{g} / \mathrm{ml}$ of propidium iodide and analyzed by flow cytometry (Coulter, XL, cytometer). Data were analyzed with FlowJo 8.7 software.

DNA Combing. Exponential growing MEL DS-19 cultures at $5 \times 10^{5}$ cells $/ \mathrm{ml}$ were pulse labelled for 20 minutes with $40 \mu \mathrm{M}$ IdU (Sigma), followed by a second $20 \mathrm{~min}$ pulse with $400 \mu \mathrm{M}$ CldU (Sigma). After harvest, the cells were embedded in low melting point agarose (BioRad) plugs, at a density of $4 \times 10^{5}$ cells per plug. Plugs were

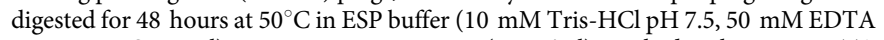
$\mathrm{pH} 8.0,1 \%$ Sarcosyl) containing proteinase $\mathrm{K}(2 \mathrm{mg} / \mathrm{ml})$, washed with $0.5 \mathrm{M}$ EDTA $\mathrm{pH} 8.0$ at $50^{\circ} \mathrm{C}$ and kept at $4^{\circ} \mathrm{C}$. For DNA extraction, plugs were washed 10 minutes with TE (10 mM Tris HCl pH 8.0/ $1 \mathrm{mM}$ EDTA pH8.0) and stained with $1.5 \mu$ YoYo (Invitrogen) in $100 \mu \mathrm{l}$ of TE for 1 hour in the dark on a rocking platform at $300 \mathrm{rpm}$ Plugs were melted in $50 \mathrm{mM}$ MES pH 5.7 at $65^{\circ} \mathrm{C}$ for 1 hour and the agarose was digested overnight with $\beta$-agarase (BioLabs), 3 units per plug. Combing of DNA fibres was performed at Philippe Pasero's lab as previously described (Pasero et al, 2002). Briefly, silanized coverslips (Schwob's lab) were incubated in DNA solutions for 15 minutes at room temperature and removed from the reservoir at a constant speed of $300 \mu \mathrm{m} / \mathrm{s}$. To fix the fibres the coverslips were baked for 2 hours at $65^{\circ} \mathrm{C}$.

Immunofluorescent Detection. Slides were dehydrated for 5 minutes in successive ethanol baths of $70 \%, 90 \%$ and $100 \%$, denatured in $1 \mathrm{M} \mathrm{NaOH}$ for 25 minutes, neutralized with PBS and blocked in 1x PBS, 1\% BSA and $0.1 \%$ TritonX100 for 60 minutes. Hybridization was carried-out in a humid chamber at $37^{\circ} \mathrm{C}$. IdU and CldU signals were developed by sequential incubations with antibodies separated by PBS washings, as follows: primary antibody mix, 1/20 Mouse Anti-BrdU clone B44, (Becton Dickinson) (anti-IdU) and Rat Anti-BrdU clone BU1/75, (AbCys SA) (antiCldU) for 45 minutes, secondary antibody mix, 1/50 Goat anti-Mouse Alexa 546 (Molecular Probes) and Goat anti-Rat Alexa 488 (Molecular Probes) for 30 minutes. DNA detection was developed by $1 / 100$ Mouse anti ssDNA (Chemicon) for 30 minutes and Goat anti- Mouse IgG2a Alexa 647 (Molecular Probes) for 30 minutes.

Image acquisition and analysis. Image acquisition was performed with a Leica DM6000B microscope equipped with a CoolSNAP HQ CCD camera and controlled with MetaMorph (Roper Scientific). On images acquired with this CCD camera and a $40 \mathrm{x}$ objective, 1 pixel $=340 \mathrm{pb}$. IdU and CldU tracks were measure manually with Photoshop (Adobe CS3) and data were transferred to an Excel Spreadsheet (Microsoft). Statistical analysis of CldU track length and inter-origin distance was performed with Prism 4.0 (GraphPad).

Indirect immunofluorescent staining. MEL DS-19 cells were fixed with 70\% cold ethanol overnight at $4{ }^{\circ} \mathrm{C}$. Cells were permeabilised with $0,1 \%$ Triton X-100 in PBS for 30 minutes, blocked in $10 \%$ normal goat serum in PBS at room temperature for 1 hour. Primary and secondary antibody incubations were carried out in blocking buffer at room temperature for 1 hour each one and washes were performed using PBS. The primary antibodies used were: anti-HP1 $\alpha$ (clone $15.19 \mathrm{~s} 2$, \#05-689, Upstate, USA) at a dilution of $1: 200$ and anti-phospho-Histone H2A.X Ser 139, (clone JBW30, 05-636 Millipore) at a dilution of 1:500. In both cases the secondary antibody 
DAPI

a

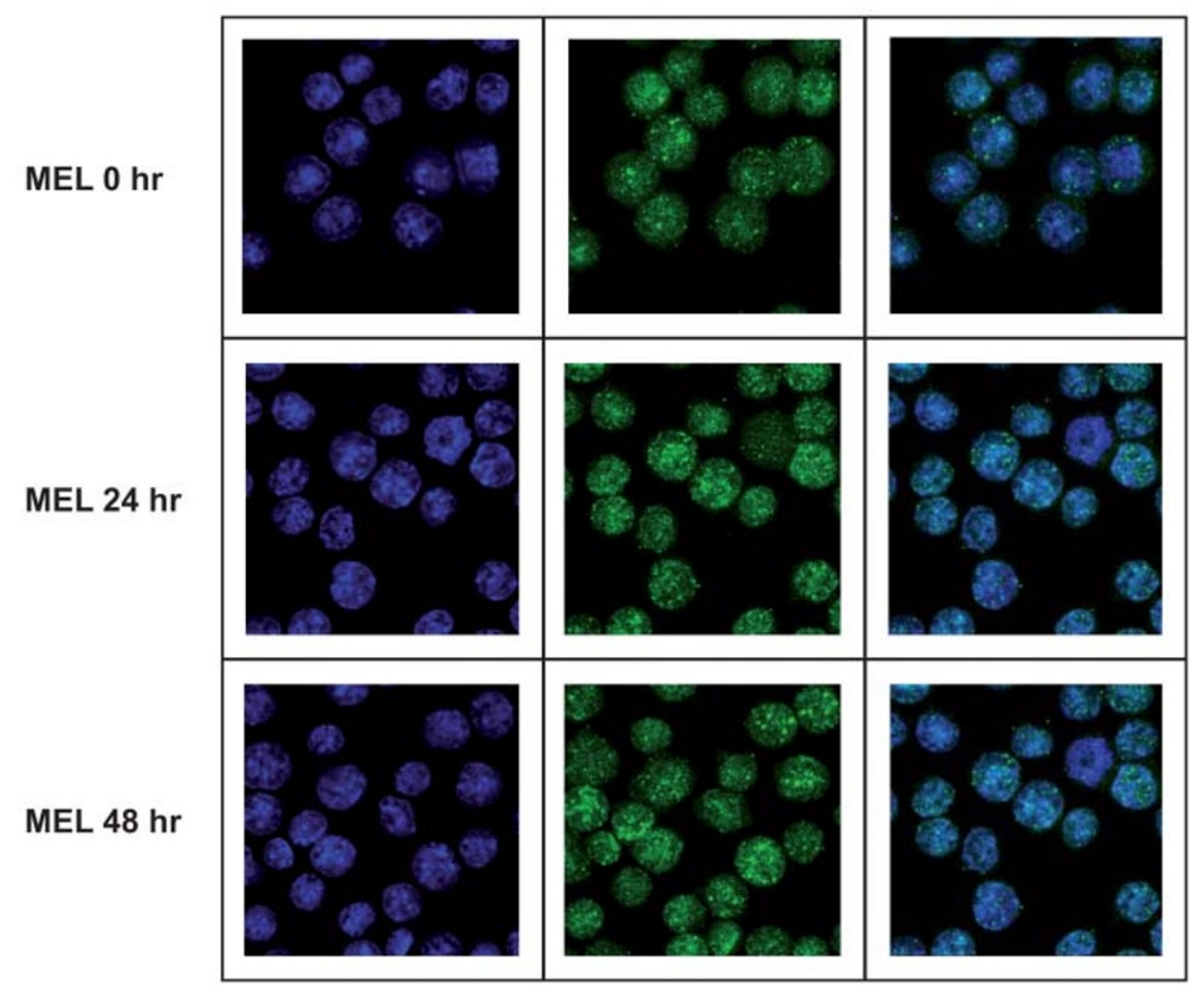

b

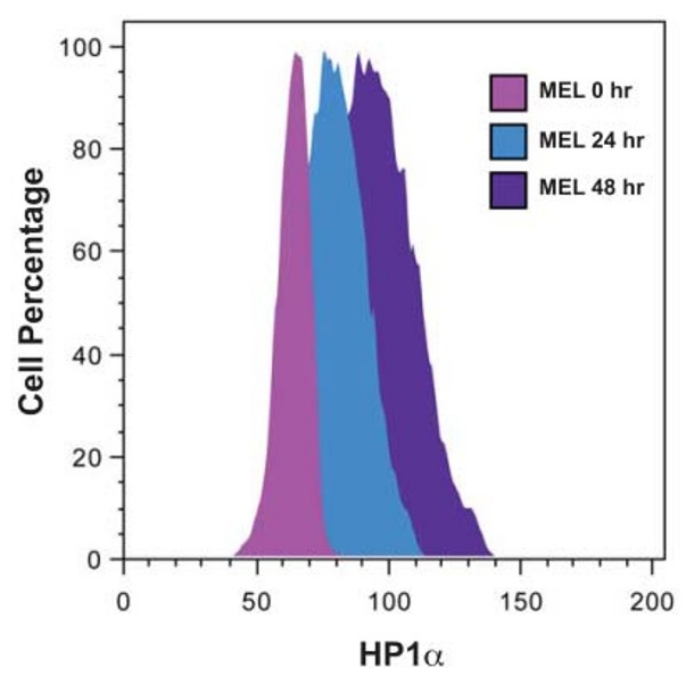

Figure $4 \mid$ Heterochromatinization, as measured by the amount of HP1 $\alpha$, increases continuously during MEL terminal differentiation. (a) MEL-DS19 cells untreated or treated with HMBA for 24 or $48 \mathrm{hr}$ were first stained with DAPI (blue), incubated with anti-HP1 $\alpha$ followed by the secondary antibody, anti-mouse Alexa 488 (green) as described in Material and Methods. (b) MEL-DS19 cells treated with anti-HP1 $\alpha$ followed by the secondary antibody, anti-mouse Alexa 488 and analysed by flow cytometry. The panel shows the percentage of cells with different levels of HP1 $\alpha$ in each case.

used was anti-mouse Alexa 488 (Molecular Probes) at a dilution of $1: 400$ and $1: 1000$, respectively. Cells were counterstained with DAPI (4_6-diamidino-2-phenylindole, $0.2 \mu \mathrm{g} / \mathrm{ml}$, Sigma). Cells were spinned to slides and mounted with Pro Long Gold (Invitrogen). Images of immunostained cell nuclei were aquired with a Leica TCS SP5 Confocal Laser microscope equipped with a $63 \mathrm{x}$ immersion oil objective. Pictures were processed using Adobe Photoshop 4.0 software.

HP1 $\alpha$ flow cytometry. MEL DS-19 cells were fixed with $70 \%$ cold ethanol overnight at $4^{\circ} \mathrm{C}$. Cells were permeabilised with $0,1 \%$ Triton X-100 in PBS for 30 minutes and incubated with anti-HP1 $\alpha$ at a dilution of 1:500 for 1 hour at room temperature. After three washes with PBS cells were incubated with the secondary antibody: antimouse Alexa 488 (Molecular Probes) at a dilution of 1:1000 for 1 hour at room temperature. Cells were washed with PBS twice and incubated with $20 \mu \mathrm{g} / \mathrm{ml}$ of propidium iodide and $100 \mu \mathrm{g} / \mathrm{ml}$ of RNAsa for 30 minutes at room temperature.
Samples were analysed by flow cytometry (Coulter, XL, cytometer) and data was analyzed with FlowJo 8.7 software.

Ribonucleotide reductase expression analysis. Total RNA was extracted from cells using Trizol reagent First-strand cDNA was synthesized from $5.0 \mu \mathrm{g}$ of total RNA using the Superscript II (Invitrogen) in a final volume of $20 \mu \mathrm{l}$ with $0.5 \mu \mathrm{g}$ of Oligo dT (Invitrogen), 20 units of SUPERase In RNase Inhibitor (Ambion) and 200 units of Superscript II reverse transcriptase. The reaction mixture was incubated at $42^{\circ} \mathrm{C}$ for 50 min. Quantitative real time PCR was carried out in iQ5 system (Bio-Rad). The reaction mixture of $20 \mu \mathrm{l}$ consisted of $1 \times$ iQ SYBR Green Supermix (Bio-Rad), $1 \mu \mathrm{l}$ cDNA and $0.2 \mu \mathrm{M}$ of each primer. The PCR protocol was: $95^{\circ} \mathrm{C}$ for $5 \mathrm{~min}$, followed by 50 cycles of $95^{\circ} \mathrm{C}$ for $30 \mathrm{~s}$ and $60^{\circ} \mathrm{C}$ for $30 \mathrm{~s}$. The following primers were used: RNRM1, 5'-CCTGGTCTGGACGAGGTCT-3' (forward) and 5'-CGACCCTGCTTCTCGTAACT-3' (reverse). RNRM2, 5' -TTTCTTTGCAGCGAGTGATG-3' 
(forward) and 5' -CGGGCCTCTGTAACTTGAAC-3' (reverse). GAPDH, 5'-GGGTTCCTATAAATACGGACTGC- $3^{\prime}$ (forward) and $5^{\prime}$-CCATTTTGTCTACGGGACGA-3' (reverse). Beta-Actin, 5' -CTAAGGCCAACCGTGAAAAG-3' (forward) 5'-ACCAGAGGCATACAGGGACA-3' (reverse). Relative gene-expression quantification method was used to calculate the fold change of mRNA expression according to the comparative $\mathrm{C}_{\mathrm{t}}$ method using GAPDH and $\beta$-actin as endogenous controls. Final results were determined as follows: $2^{-(\Delta \mathrm{Ct} \text { sample }-\Delta \mathrm{Ct} \text { control) }}$, where $\Delta \mathrm{C}_{\mathrm{t}}$ values of the control and sample were determined by subtracting the $C_{t}$ value of the target gene, RNR, from the value of the housekeeping gene: GAPDH and $\beta$-actin.

1. Aladjem, M. I. Replication in context: dynamic regulation of DNA replication patterns in metazoans. Nat Rev Genet 8, 588-600 (2007).

2. Zhou, J. et al. Changes in replication, nuclear location, and expression of the Igh locus after fusion of a pre-B cell line with a T cell line. J Immunol 175, 2317-2320 (2005).

3. DePamphilis, M. L. Eukaryotic DNA replication origins: Reconciling disparate data. Cell 114, 274-275 (2003).

4. Mechali, M. DNA replication origins: from sequence specificity to epigenetics. Nat Rev Genet 2, 640-645 (2001).

5. Norio, P. DNA replication: the unbearable lightness of origins. EMBO Rep 7, 779781 (2006).

6. Hiratani, I. et al. Global reorganization of replication domains during embryonic stem cell differentiation. PLoS Biol 6, e245 (2008).

7. Norio, P. et al. Progressive activation of DNA replication initiation in large domains of the immunoglobulin heavy chain locus during B cell development. Mol Cell 20, 575-587 (2005).

8. Cardoso, M. C., Leonhardt, H. \& Nadal-Ginard, B. Reversal of terminal differentiation and control of DNA replication: cyclin A and Cdk2 specifically localize at subnuclear sites of DNA replication. Cell 74, 979-992 (1993).

9. Zhu, L. \& Skoultchi, A. I. Coordinating cell proliferation and differentiation. Curr Opin Genet Dev 11, 91-97 (2001)

10. Marks, P. A. \& Rifkind, R. A. Erythroleukemic differentiation. Аnпи Rev Biochem 47, 419-448 (1978)

11. Friedman, E. A. \& Schildkraut, C. L. Terminal differentiation in cultured Friend erythroleukemia cells. Cell 12, 901-913 (1977).

12. Matushansky, I., Radparvar, F. \& Skoultchi, A. I. Reprogramming leukemic cells to terminal differentiation by inhibiting specific cyclin-dependent kinases in G1. Proc Natl Acad Sci USA 97, 14317-14322 (2000).

13. Rao, G., Rekhtman, N., Cheng, G., Krasikov, T. \& Skoultchi, A. I. Deregulated expression of the PU.1 transcription factor blocks murine erythroleukemia cell terminal differentiation. Oncogene 14, 123-131 (1997).

14. Vanegas, N. et al. Differential expression of Ran GTPase during HMBA-induced differentiation in murine erythroleukemia cells. Leukemia Res 27, 607-615 (2003).

15. Fernandez-Nestosa, M. J., Hernandez, P., Schvartzman, J. B. \& Krimer, D. B. PU.1 is dispensable to block erythroid differentiation in Friend erythroleukemia cells. Leukemia Res 32, 121-130 (2008)

16. García-Sacristán, A., Fernández-Nestosa, M. J., Hernández, P., Schvartzman, J. B. \& Krimer, D. B. Protein kinase clk/STY is differentially regulated during erythroleukemia cell differentiation: a bias toward the skipped splice variant characterizes postcommitment stages. Cell Res 15, 495-503 (2005).

17. Krimer, D. B., Cheng, G. \& Skoultchi, A. I. Induction of H3.3 replacement histone mRNAs during the precommitment period of murine erythroleukemia cell differentiation. Nucleic Acids Res 21, 2873-2879 (1993).

18. Stopka, T., Amanatullah, D. F., Papetti, M. \& Skoultchi, A. I. PU.1 inhibits the erythroid program by binding to GATA-1 on DNA and creating a repressive chromatin structure. EMBO Journal 24, 3712-3723 (2005).

19. Prasanth, S. G., Shen, Z., Prasanth, K. V. \& Stillman, B. Human origin recognition complex is essential for HP1 binding to chromatin and heterochromatin organization. Proc Natl Acad Sci USA 107, 15093-15098 (2010).

20. Gregoire, D., Brodolin, K. \& Mechali, M. HoxB domain induction silences DNA replication origins in the locus and specifies a single origin at its boundary. $E M B O$ Rep 7, 812-816 (2006).

21. Schultz, S. et al. Single molecule analysis reveals changes in the DNA replication program for the POU5F1 locus upon hESC differentiation. Mol Cell Biol 30, 4521-4534 (2010).

22. Levy, J., Terada, M., Rifkind, R. A. \& Marks, P. A. Induction of erythroid differentiation by dimethylsulfoxide in cells infected with Friend virus: relationship to the cell cycle. Proc Natl Acad Sci USA 72, 28-32 (1975).

23. Khochbin, S., Chabanas, A., Albert, P., Albert, J. \& Lawrence, J. J. Application of bromodeoxyuridine incorporation measurements to the determination of cell distribution within the S phase of the cell cycle. Cytometry 9, 499-503 (1988).

24. Anglana, M., Apiou, F., Bensimon, A. \& Debatisse, M. Dynamics of DNA replication in mammalian somatic cells: Nucleotide pool modulates origin choice and interorigin spacing. Cell 114, 385-394 (2003).

25. Conti, C. et al. Inhibition of histone deacetylase in cancer cells slows down replication forks, activates dormant origins, and induces DNA damage. Cancer Res 70, 4470-4480 (2010).

26. Courbet, S. et al. Replication fork movement sets chromatin loop size and origin choice in mammalian cells. Nature 455, 557-560 (2008)

27. Lebofsky, R., Heilig, R., Sonnleitner, M., Weissenbach, J. \& Bensimon, A. DNA replication origin interference increases the spacing between initiation events in human cells. Mol Biol Cell 17, 5337-5345 (2006).
28. Lemaitre, J. M., Danis, E., Pasero, P., Vassetzky, Y. \& Mechali, M. Mitotic remodeling of the replicon and chromosome structure. Cell 123, 787-801 (2005)

29. Pasero, P., Bensimon, A. \& Schwob, E. Single-molecule analysis reveals clusterin and epigenetic regulation of replication origins at the yeast rDNA locus. Genes Dev 16, 2479-2484 (2002)

30. Chabes, A. \& Stillman, B. Constitutively high dNTP concentration inhibits cell cycle progression and the DNA damage checkpoint in yeast Saccharomyces cerevisiae. Proc Natl Acad Sci USA 104, 1183-1188 (2007).

31. Reichard, P. Interactions between deoxyribonucleotide and DNA synthesis. Annu Rev Biochem 57, 349-374 (1988).

32. Mathews, C. K. \& Sinha, N. K. Are DNA precursors concentrated at replication sites? Proc Natl Acad Sci USA 79, 302-306 (1982).

33. Sanvisens, N., Bano, M. C., Huang, M. \& Puig, S. Regulation of ribonucleotide reductase in response to iron deficiency. Mol Cell 44, 759-769 (2011).

34. Petermann, E., Orta, M. L., Issaeva, N., Schultz, N. \& Helleday, T. Hydroxyureastalled replication forks become progressively inactivated and require two different RAD51-mediated pathways for restart and repair. Molecular cell 37, 492-502 (2010).

35. Blow, J. J., Ge, X. Q. \& Jackson, D. A. How dormant origins promote complete genome replication. Trends Biochem Sci 36, 405-414 (2011).

36. Patel, P. K. et al. The Hsk1(Cdc7) replication kinase regulates origin efficiency. Mol Biol Cell 19, 5550-5558 (2008).

37. Gay, S. et al. Nucleotide supply, not local histone acetylation, sets replication origin usage in transcribed regions. EMBO Rep 11, 698-704 (2010).

38. Ge, X. Q., Jackson, D. A. \& Blow, J. J. Dormant origins licensed by excess Mcm2-7 are required for human cells to survive replicative stress. Genes \& Development 21, 3331-3341 (2007).

39. Karnani, N. \& Dutta, A. The effect of the intra-S-phase checkpoint on origins of replication in human cells. Genes \& Development 25, 621-633 (2011).

40. Kawabata, T. et al. Stalled fork rescue via dormant replication origins in unchallenged $S$ phase promotes proper chromosome segregation and tumor suppression. Molecular cell 41, 543-553 (2011).

41. Klotz-Noack, K. \& Blow, J. J. A role for dormant origins in tumor suppression. Molecular cell 41, 495-496 (2011).

42. Kunnev, D. et al. DNA damage response and tumorigenesis in Mcm2-deficient mice. Oncogene 29, 3630-3638 (2010).

43. Woodward, A. M. et al. Excess Mcm2-7 license dormant origins of replication that can be used under conditions of replicative stress. J Cell Biol 173, 673-683 (2006).

44. Skoog, L. \& Nordenskjold, B. Effects of hydroxyurea and 1-beta-Darabinofuranosyl-cytosine on deoxyribonucleotide pools in mouse embryo cells. Eur J Biochem 19, 81-89 (1971).

45. Leach, T. J., Chotkowski, H. L., Wotring, M. G., Dilwith, R. L. \& Glaser, R. L. Replication of heterochromatin and structure of polytene chromosomes. Mol Cell Biol 20, 6308-6316 (2000).

46. Takebayashi, S. et al. Regulation of replication at the R/G chromosomal band boundary and pericentromeric heterochromatin of mammalian cells. Exp Cell Res 304, 162-174 (2005).

47. James, T. C. \& Elgin, S. C. Identification of a nonhistone chromosomal protein associated with heterochromatin in Drosophila melanogaster and its gene. Mol Cell Biol 6, 3862-3872 (1986).

48. Rimmele, P. et al. Spi-1/PU.1 oncogene accelerates DNA replication fork elongation and promotes genetic instability in the absence of DNA breakage. Cancer research 70, 6757-6766 (2010).

\section{Acknowledgements}

We acknowledge María-Luisa Martínez-Robles, María-José Fernández-Nestosa, Virginia López and María Rodríguez for their suggestions and support during the course of this study. We also acknowledge Adela Calvente-Arroyo and José Luis Barbero Esteban for antibodies and advice in immunocytochemistry. This work was sustained by grants BFU2008-00408/BMC and BFU2011-22489 to JBS from the Spanish Ministerio de Ciencia e Innovación.

\section{Author contribution Statement}

$\mathrm{M}^{\mathrm{a}}$ Estefanía Monturus performed all the experimental work. Olivier Ganier and Marcel Mechali designed, participated and advised all the experiments involving DNA combing. Pablo Hernández participated in the analysis of cell flow cytometry data. Jorge B. Schvartzman and Dora B. Krimer designed the experiments, analysed the results and wrote the manuscript.

\section{Additional information}

Supplementary information accompanies this paper at http://www.nature.com/ scientificreports

Competing financial interests: The authors declare no competing financial interests.

License: This work is licensed under a Creative Commons

Attribution-NonCommercial-ShareAlike 3.0 Unported License. To view a copy of this license, visit http://creativecommons.org/licenses/by-nc-sa/3.0/

How to cite this article: Monturus et al. DNA Replication Fading As Proliferating Cells Advance in Their Commitment to Terminal Differentiation. Sci. Rep. 2, 279; DOI:10.1038/ srep00279 (2012) 